\title{
DISPONIBILIDAD, INCERTIDUMBRE Y CADENA DE FALLO EN MANTENIMIENTO
}

\section{AVAILABILITY, UNCERTAINTY AND MAINTENANCE FAILURE CHAIN}

Francisco Javier Cárcel Carrasco ${ }^{1}$

1. Doctor Ingeniero Industrial. Universitat Politècnica de València. Camino de Vera $S / N$, 46022, Valencia, España. E-mail: fracarc1@csa.upv.es 


\section{RESUMEN}

En este artículo, se analiza la actividad de mantenimiento, en lo referente a la disponibilidad, la incertidumbre y la fiabilidad, cuya misión es conseguir una utilización óptima de los activos productivos, manteniéndolos en el estado que requiere una producción eficiente. El objetivo de la actividad de mantenimiento es conseguir de forma eficiente los valores requeridos de disponibilidad, conviene reflexionar sobre el concepto de disponibilidad, los factores clave que influyen en ella y cómo se plantea en la actualidad su conocimiento. Hay que tener en cuenta la definición de fallo como una desviación de una condición original de un equipo o sistema, cuyo funcionamiento pasa a ser catalogado como insatisfactorio para un utilizador concreto. La determinación de que el funcionamiento es insatisfactorio depende de la evaluación previa que se realice de las consecuencias del fallo en un contexto operativo determinado.

\section{ABSTRACT}

This article discusses the maintenance activity, in relation to the availability, uncertainty and reliability, whose mission is to achieve an optimal use of productive assets, keeping them in the State that requires an efficient production. Maintenance activity aims to achieve efficiently the required values of availability, should reflect on the concept of availability, the key factors that influence it and how their knowledge at present arises. It should take into account the definition of failure as a deviation from an original condition of an equipment or system, which happens to be rated as unsatisfactory for a particular user. The determination that the performance is unsatisfactory depends on ex-ante evaluation carried out of the consequences of failure in a given operating context.

\section{PALABRAS CLAVE}

Mantenimiento industrial; Disponibilidad; Incertidumbre; Cadena de fallo.

\section{KEY WORDS}

Industrial maintenance; Availability; Uncertainty; Fault string. 


\section{INTRODUCCIÓN}

Cualquier elemento de la cadena de fallo es observable e identificable a través de unos síntomas o manifestaciones diversas (vibración, ruido o zumbido, olor, calor o frío, aumento o disminución de la visibilidad, humo, humedad, polvo, abrasión o corrosión, desgaste, rotura, desprendimiento, etc.). Estos síntomas son claves a la hora de identificar la cadena.

Dado que el objetivo de la actividad de mantenimiento es conseguir de forma eficiente los valores requeridos de disponibilidad, conviene reflexionar sobre el concepto de disponibilidad, los factores clave que influyen en ella y cómo se plantea en la actualidad su conocimiento.

El objetivo básico de un programa de mantenimiento es conseguir la disponibilidad efectiva de la planta. Los organismos europeos de normalización han fijado definiciones sobre la disponibilidad. De entre ellas, destaca la propuesta por la British Standards Institution (British Standars, 1964) por su sencillez intuitiva:

"Disponibilidad de un ítem en un período determinado es la fracción de dicho período durante la cual es capaz de realizar una función específica a un determinado nivel de rendimiento". 


\section{LA DISPONIBILIDAD EN MANTENIMIENTO}

Una tal definición operativa de Mantenimiento Industrial podría ser el conjunto de técnicas que tienen por objeto conseguir una utilización óptima de los activos productivos, manteniéndolos en el estado que requiere una producción eficiente.

El objetivo básico de un programa de mantenimiento es conseguir la disponibilidad efectiva de la planta. Esto requiere:

- Alcanzar el nivel de disponibilidad requerida en equipos e instalaciones.

- Hacerlo al menor coste posible.

- Incorporar otros objetivos como menor tiempo de actuación o elevada calidad del trabajo realizado.

Para conseguir estos objetivos se hará preciso alcanzar otros como los siguientes:

- Evaluar los requerimientos y capacidades técnicas de los equipos e instalaciones. Esta información influirá en el diseño o selección de los mismos y en la determinación de las condiciones de operación.

- Identificar los factores o causas que impiden al sistema alcanzar los niveles de disponibilidad especificados, entre otros, los insuficientes niveles de fiabilidad de diseño u operativa o de mantenibilidad.

- Proponer acciones eficientes encaminadas a alcanzar los niveles de disponibilidad objetivo.

- Determinar y evaluar las tecnologías y técnicas de detección, diagnóstico, verificación y prueba, y de restauración de las condiciones iniciales, incluyendo los correspondientes procedimientos.

- Seguir y controlar la aplicación correcta de las técnicas y procedimientos, y de la actividad de mantenimiento en general.

- Recomendar acciones de mejora continua de la disponibilidad y de sus factores causales.

- Integrar la actividad y función de mantenimiento con el resto de funciones que intervienen en el ciclo de vida del sistema, evaluando su esperanza de vida y, en consecuencia, la rentabilidad a través de la actualización de los flujos de efectivo.

Significando "ítem" todo elemento, equipo o sistema susceptible de ser considerado, examinado y comprobado por separado.

De esta definición han de resaltarse dos aspectos fundamentales. En primer lugar, el estado de "disponible" no implica necesariamente que un ítem esté funcionando en el instante o período considerado, sino que se encuentre en la situación de "apto para funcionar". Además, aunque un ítem se encuentre "funcionando", puede estar no "disponible" sino funciona de acuerdo con las especificaciones requeridas. 
Aunque podrían generarse dificultades conceptuales y de captación de la información, la consideración de determinados estados intermedios, desde funcionar adecuadamente a estar averiados (como sería el caso de tener que producir a baja capacidad, o con un consumo energético excesivo, o con alguna deficiencia de calidad), puede mejorar sensiblemente el conocimiento del comportamiento del equipo en base a la experiencia sobre variados escenarios. Esto ha de añadir necesariamente un conocimiento específico valioso sobre los diferentes modos de fallo. 


\section{LA INCERTIDUMBRE EN MANTENIMIENTO}

La incertidumbre sobre el adecuado comportamiento de un equipo o sistema durante su ciclo de vida puede originarse en cualquier etapa. En general, puede afirmarse que dicha incertidumbre aumenta hasta las últimas etapas del ciclo, donde ya los factores que inciden sobre la incertidumbre se estabilizan, y el control y la predicción parten de experiencias y conocimiento base contrastado (Figura 1). Algunas de las fuentes de incertidumbre más comunes quedan reflejadas en la figura 6 Pueden clasificarse esas fuentes de incertidumbre en los siguientes grupos:

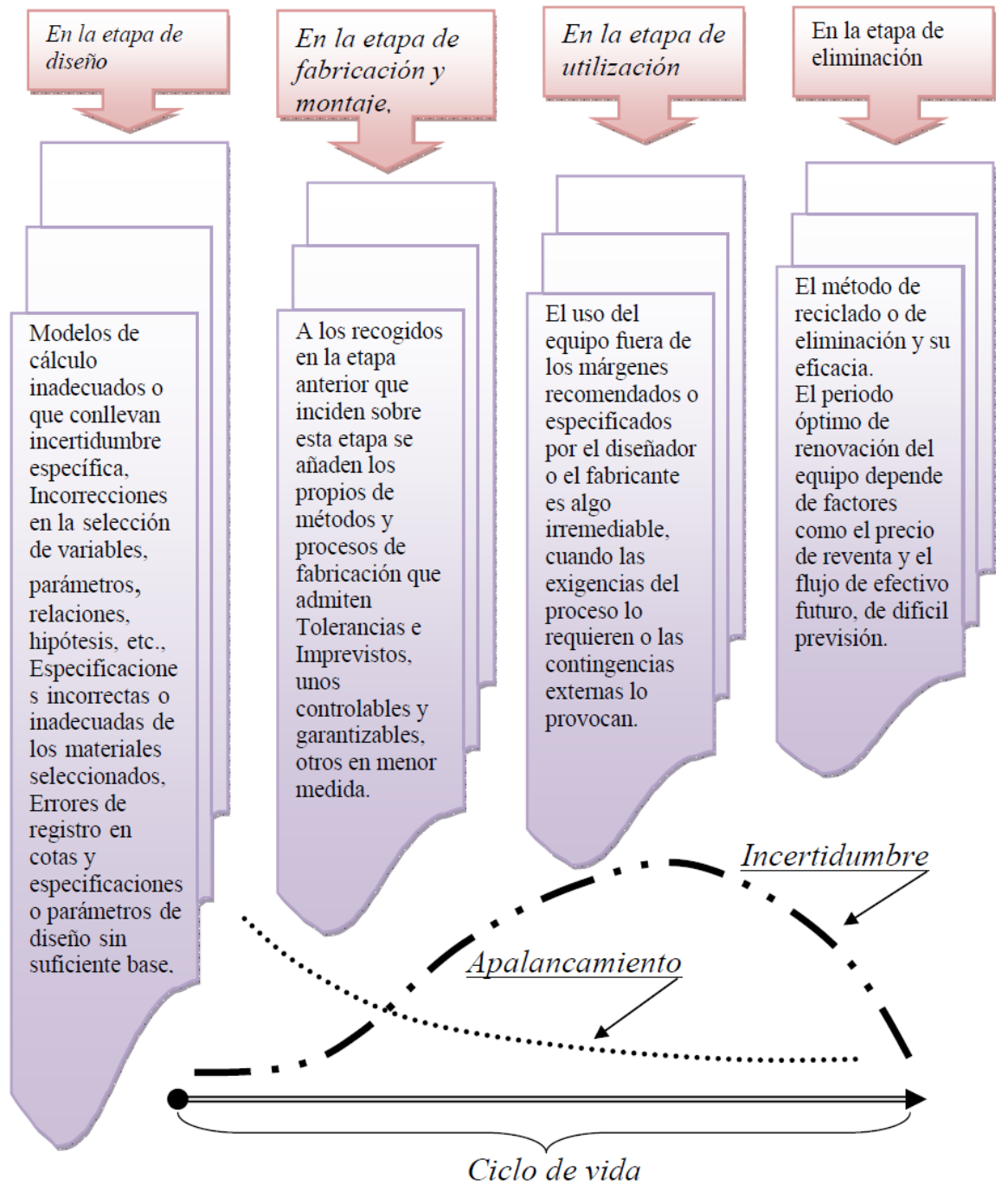

Figura 1. Fuentes de incertidumbre.

- Incertidumbre Fenomenológica.

- Incertidumbre de Determinación.

- Incertidumbre de los Planteamientos.

- Incertidumbre de la Actividad. 


\section{Incertidumbre fenomenológica}

El fenómeno de fallo suele implicar la existencia de diversas y variadas causas, poco e insuficientemente conocidas, constituyendo así un término de error (término aleatorio o residuos) en los modelos comprensivos del comportamiento al fallo de equipos, instalaciones o sistemas.

De hecho, la existencia de incertidumbre tiene origen en la insuficiencia e incompletitud del conocimiento sobre el fenómeno físico que tiene lugar y el comportamiento derivado del sistema. Señala la incapacidad por comprender e interpretar, a un nivel requerido, ese fenómeno.

La naturaleza aleatoria del fallo, en general, parece incuestionable. Si existe algún fenómeno que se produce en el ambiente de la planta industrial, que puede certificarse como estocástico, es por excelencia el del fallo.

Desde un punto de vista filosófico, parece que el origen último del fallo de sistemas físicos se puede asociar con una decisión o acción humanas. Desde el operativo, también es preciso señalar la intervención relevante del factor humano en la explicación del fallo físico, añadiendo, sin duda, así, nuevas dosis de incertidumbre al tratarse de un sistema mucho más complejo, imprevisible $y$, en definitiva, con un comportamiento menos regular que la máquina.

Además de las causas humanas, es preciso hacer mención de las causas naturales, imprevistos y catástrofes de diversa y variada índole, que se encuentran con frecuencia formando parte de la cadena de fallo. En su propia esencia llevan implícito la imprevisibilidad y dificultad de comprensión y evaluación y, por tanto, su carácter eminentemente aleatorio. En general, puede afirmarse que los estados límite, que se presentan en los estadios últimos del proceso de fallo, suelen conducir a explicaciones o modelos estocásticos.

\section{Incertidumbre de determinación}

Se ha abundado también en las elevadas dosis de incertidumbre que se dan en la definición o determinación del fallo, dado que se trata de una decisión con evidentes aspectos discrecionales, sometidos a avatares operacionales o de interés para la toma de decisiones. Dada la dificultad existente para determinar unívocamente, en cada caso, lo que procede definir como fallo, se acude a buscar o solicitar características que entronquen con ventajas operacionales (facilidad de descripción, aspectos observables directamente, etc.) u orientadas a la toma de decisiones, aspectos ambos con indudables dosis de aleatoriedad y discrecionalidad. Además, el establecimiento de los umbrales de fallo es algo intrínsecamente voluntario y dependiente de las variables de negocio, alejado, por tanto, de consideraciones absolutamente científicas o modelables.

La evaluación y valoración de la existencia y evolución del fallo son considerablemente volátiles, discrecionales y dependen de consideraciones basadas en una experiencia y conocimientos incompletos, fraccionados y de difícil aprehensión. Esta incapacidad por 
comprender la totalidad de lo que ocurre o puede llegar a ocurrir conduce a planteamientos de tipo aleatorio.

\section{Incertidumbre de los planteamientos}

A partir de los hechos anteriores: carácter aleatorio del fenómeno y la determinación del fallo, lógicamente se derivan planteamientos que conducen a modelos (delimitación o simplificación de la realidad) de carácter aleatorio.

Los modelos de fiabilidad representan simplificaciones importantes, pero necesarias, que derivan en elevados niveles de incertidumbre, en el ámbito de las variables y sus relaciones.

\section{Incertidumbre en la actividad}

La actividad de mantenimiento: detección, diagnóstico y reparación en actuaciones de carácter correctivo, preventivo o predictivo, está sometida a contingencias diversas, se produce en escenarios complejos y diferentes y no es fácil que se presenten regularidades que puedan conducir a una actividad fácilmente planificable y controlable.

En definitiva, parece poder establecerse sin dificultad que la incertidumbre es una de las características esenciales del mantenimiento, que hacen de éste una de las funciones o actividades de la planta industrial más complejas y difíciles de conocer. Efectivamente, esa incertidumbre no deja de ser una medida de la imperfección del conocimiento sobre este sujeto. La cuestión no parece ser tanto la eliminación de la varianza no explicada, como la de encontrar el nivel óptimo de esa varianza, ya que los procesos de generación y aplicación de conocimientos implican costes que deben ser considerados.

\section{Experimentalidad y modelado de sistemas.}

De lo determinado con anterioridad: la existencia de elevados niveles de incertidumbre en lo relativo al proceso y a la cadena del fallo, se puede inducir sin dificultad la necesidad de un enfoque complementario al exclusivamente científico y que es el experimental. Modelos teóricos y experimentales se complementan para tratar de ofrecer conocimiento válido, en un campo en el que, como se ha señalado, abundan los imprevistos y la complejidad de las relaciones causa-efecto (Weber et al. 2006; Yongli et al., 2006; López et al., 2010; Levitin et al., 2003).

La experiencia derivada de la observación y del ensayo constituye un pilar básico del sistema de generación, transmisión, conservación y aplicación del conocimiento. El experimento, aún con bases científicas, suele ser desarrollado en la planta, toda vez que los modos de fallo contienen características diferenciales, difícilmente reproducibles en laboratorio. Además, como se ha señalado, es preciso considerar los costes derivados del análisis, lo que en general inclina éste hacia el planteamiento de ensayos en planta. La validez local de los resultados, lo que acota el carácter absolutamente científico del análisis no oculta que en la 
mayoría de los casos, la complementariedad del análisis estrictamente científico con el experimental, constituye la alternativa de elección en la planta industrial. Sin embargo, esa localidad o especificidad de los experimentos en relación con el fallo, sus causas y consecuencias, no puede ocultar que el equipo, máquina o instalación están integrados con otros muchos, en un sistema o proceso de producción-distribución. De forma similar, las piezas, componentes o conjuntos están integrados, constituyendo los equipos y no es fácil aislar su comportamiento del de otras partes vecinas, con las que comparten objetivos, esquemas y ligaduras en el funcionamiento.

Se hace también difícil deslindar y aislar el comportamiento presente del habido en otras fases del ciclo de vida, aunque así se haga en los modelos markovianos y semimarkovianos, con la hipótesis de desmemoria. En general, la aproximación sistémica parece, en consecuencia, la más adecuada a la hora de modelar y especificar el comportamiento de equipos y máquinas, en cuanto al fallo.

Es decir, que si la exportabilidad de los modelos experimentales de mantenimiento es limitada, la expansión de esos modelos viene en cambio de la consideración del ámbito del proceso de fabricación y sus relaciones con las variables de fallo; esto es, de la consideración del modelado de sistemas a partir del diseño y elaboración de experimentos.

Es preciso añadir que, en muchos supuestos, se complementa el planteamiento matemático con el lógico, especialmente en los logigramas y árboles de decisión binaria (Figura 2).

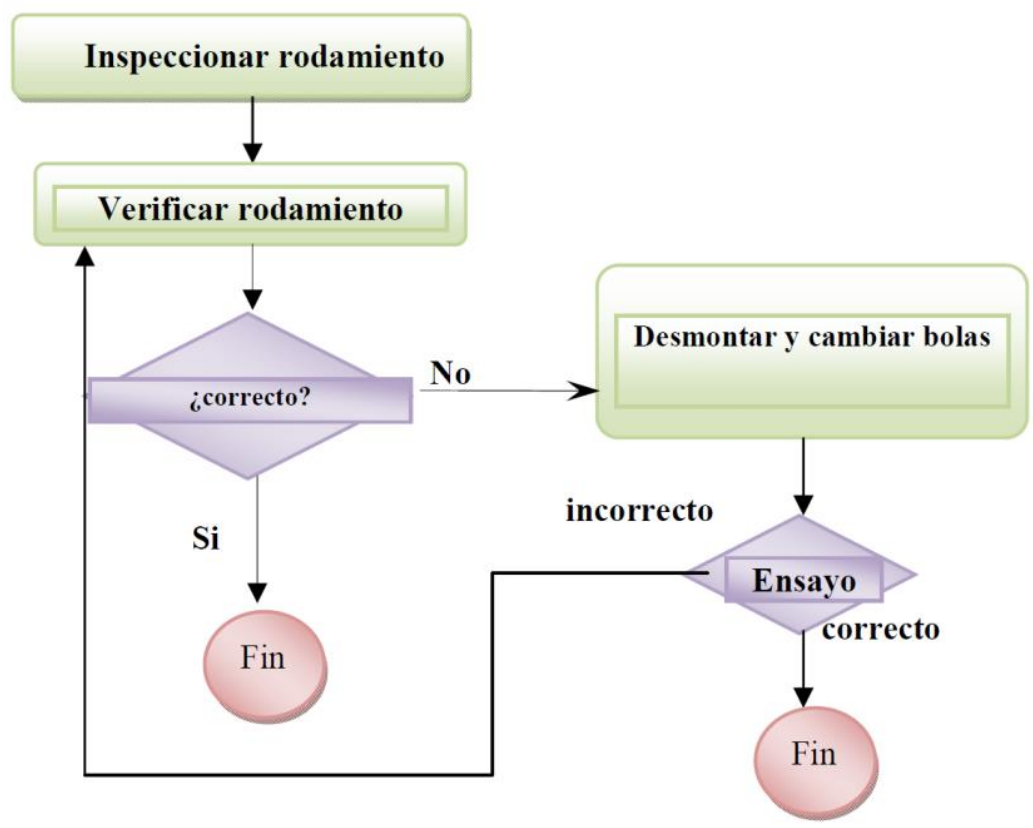

Figura 2. Árbol de decisión binaria. Fuente: Elaboración propia. 


\section{EL PROCESO DE FALLO EN MANTENIMIENTO}

En principio, y a los efectos de la función de mantenimiento industrial, fallo, en el sentido que se le asigna habitualmente, significa que un componente o un sistema no satisface o no funciona de acuerdo con la especificación (Bejar, 1974; Beltrán, 1987). Queda por tanto claro, que fallo no equivale necesariamente a parada, interrupción del funcionamiento del equipo, o no-desempeño absoluto de la función. Cualquier incidencia relativa al estado físico del equipo, que conlleva el incumplimiento de las especificaciones que debe cumplir en relación con la función, puede ser señalada como fallo.

En el estudio del proceso del fallo se relaciona los aspectos de confiabilidad y calidad en el servicio prestado por mantenimiento (IEE Std 493, 2007; Koval et al., 2003; Wang et al., 2004; Yañez et al., 2003, Cacique, 2007; Baeza et al., 2003), y se hace posible establecer nuevos indicadores que permitan estimar el nivel de seguridad de dichos sistemas, en los cuales se describa el impacto sobre la infraestructura y los riesgos asociados (McGranaghan, 2007, Sexto, 2005).

En este sentido, fallo parece asociarse a un estado del equipo o sistema que le impide cumplir con lo que se le requiere. Pero de la misma definición operativa parece desprenderse que más que de un estado único, se trata de una sucesión de estados o proceso que desemboca en una anomalía (o estado anómalo) relativa al incumplimiento de las especificaciones de funcionamiento. Lo relevante a todos los efectos es el proceso más que el estado o estados finales anómalos, ya que aquel explica las causas u orígenes, la evolución, las manifestaciones y efectos consiguientes. El mecanismo causa-efecto es el que se sitúa en la esencia del mantenimiento.

Según la definición operativa anterior, un fallo es una desviación de una condición original de un equipo o sistema, cuyo funcionamiento pasa a ser catalogado como insatisfactorio para un utilizador concreto. La determinación de que el funcionamiento es insatisfactorio depende de la evaluación previa que se realice de las consecuencias del fallo en un contexto operativo determinado (Beltrán, 1989), ya que esas consecuencias son las que fijan la prioridad de las actividades de mantenimiento o mejoras de diseño necesarias para impedir el fallo (Ramos et al., 2007; Vásquez et al., 2007; Gómez de León, 1998). Así, se implementan acciones que reducen el riesgo de fallo, a un coste menor que el derivado de las consecuencias (de seguridad, medioambientales, operacionales, etc.) o efectos que evitan.

En relación con el proceso de fallo, es interesante considerar el modelo que lo define en los siguientes términos: el resultado de la acción entre una tensión ocasionada por el entorno y la resistencia al fallo (que varía con el tipo de material, las características de los procesos de fabricación, la edad, etc.) del equipo o sistema. La tensión y la resistencia son variables dinámicas y su interacción define el estado de fallo en cada momento.

Este modelo entiende que existe una aptitud o capacidad del equipo o sistema ante el fallo; $y$ unas causas externas o internas cuya acción se representa por una tensión que supera la resistencia. El diseño, los tratamientos térmicos o de otra índole, el rediseño o la mejora, la eliminación de causas o su apantallamiento, etc., pueden mejorar la resistencia. Existe la 
posibilidad de diseñar equipos tolerantes al fallo, que pueden soportar un funcionamiento más allá del punto en que la tensión supera la resistencia.

La aceptación de este modelo conlleva, a los efectos de definir un sistema de conocimiento que soporte la actividad de mantenimiento industrial, la captación de información útil sobre los factores de contingencia que actúan o pueden actuar sobre equipos y sistemas, y sobre las variables y parámetros que definen y determinan la capacidad de resistencia y tolerancia al fallo. Esta información es una buena herramienta para entender los modos y procesos de fallo, pero es de difícil y costosa captación, con lo que se hace preciso configurar procesos de recogida, almacenamiento y tratamiento de la información eficientes.

En la figura 3, se representa un proceso de fallo típico, tal y como se plantea en cualquier instalación industrial. Se entiende que un proceso de fallo es un proceso estocástico, representado por diversos estados por los que puede pasar el equipo o sistema, cada uno de ellos con una probabilidad determinada. En la figura se recoge, por tanto, el proceso, que comprende un estado inicial de correcto funcionamiento Ei, otro final de pérdida de función Ef y varios intermedios que representan los diversos estados de pérdida, deterioro o degradación de la función requerida Ed1, Ed2,...Edn. De un estado al siguiente, se puede pasar con una probabilidad cuya función de densidad de parámetro $\lambda$, o al anterior, con $\mu$.

En relación con el proceso de fallo o su modo de desarrollo y forma de aparición, los fallos pueden ser clasificados de muy diversas formas, así por ejemplo, se pueden clasificar en catastróficos (se trata de fallos súbitos o totales, sin manifestación previa y se relacionan con fracturas, deformaciones, agarrotamientos, etc.), y progresivos (fallos paramétricos, que se desarrollan a lo largo de un periodo de tiempo). También se pueden clasificar en dependientes e independientes, repentinos y progresivos, estables o temporales (sus causas suelen ser los regímenes y condiciones de trabajo y las vibraciones anormales, grandes desviaciones de temperatura, etc.).

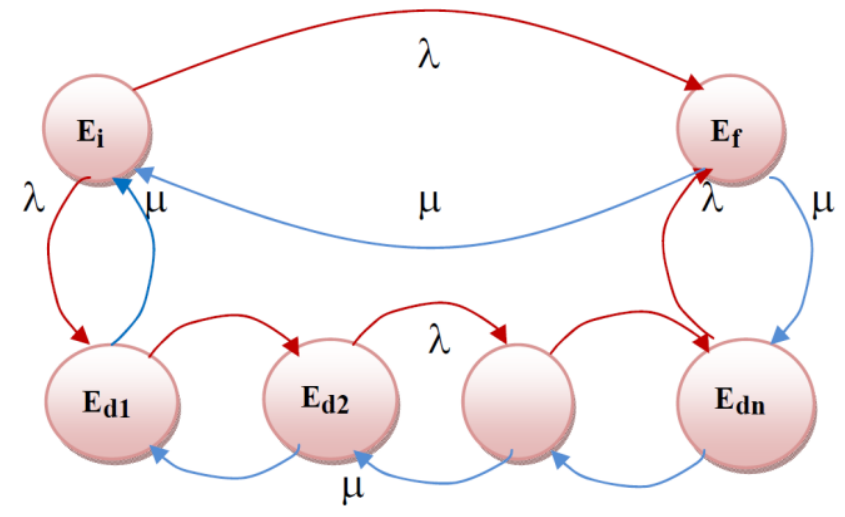

Figura 3. Representación del proceso de fallo.

Se denominan alternantes o intermitentes a los temporales muy reiterativos (que se repiten con mucha frecuencia). Resultan difíciles de descubrir y dependen de la calidad del elemento o de sus condiciones de trabajo. La existencia de fallos repetidos puede indicar un defecto de diseño o mal estado del equipo; por ello, más interesante que someterle a reparación sería el tratar de eliminar la posible causa del fallo. 
Como puede comprobarse, esta somera clasificación permite señalar la importancia que tiene la forma de aparición o manifestación del fallo en el posible diagnóstico, ya que esta componente temporal y estocástica del fallo delata muchas veces la causa o el modo de fallo y debe ser recogida como información relevante. Muestra cómo la observación, a veces relegada por las tecnologías al uso, cumple una función valiosa en mantenimiento industrial.

Así, cuando un fallo sucede, lo primero que se aprecia son sus manifestaciones, las que, analizadas convenientemente, pueden llegar a proporcionar la explicación del "modo de fallo", el cómo ha ocurrido el fallo (Bejar, 1974). Un paso más adelante representa el llegar al por qué, a la causa del fallo, a lo que se conoce como "mecánica de fallo" (Herrera et al., 1990). El proceso del fallo conlleva un determinado tiempo durante el cual se producen señales, síntomas o alteraciones, que detectadas y analizadas permiten conocer la evolución y el estado de adelanto del mismo, y el riesgo o proximidad de aparición (Delgado et al. 1994).

Un problema singular, en relación al fallo, tiene lugar cuando se observan tan pocos fallos en determinados sistemas, que resulta difícil o incluso imposible establecer su probabilidad de aparición a partir de la observación estadística. Por tanto, se tiene que recurrir, para averiguar la probabilidad de aparición, a un procedimiento analítico. Éste se basa en la descomposición del fallo del sistema en los fallos de sus componentes, para los cuales se dispone de datos observados. La observación en el ámbito de componentes es posible puesto que se utiliza la misma clase de componentes en muchos sistemas, lo cual implica un número elevado de objetos de observación, y además los componentes suelen fallar más frecuentemente que los sistemas en los cuales se utilizan.

En cualquier caso, tanto la observación, como la determinación mediante esquemas formales y el consiguiente análisis del proceso de fallo, deben tratar de dar solución a los tres problemas clásicos y fundamentales: a) origen del fallo; b) solución del fallo y c) prevención del fallo.

En relación con el primero: el conocimiento del origen del fallo, debe determinarse la cadena de fallo, presentada en este artículo como el segundo de los elementos esenciales del fallo y que se analiza en el apartado siguiente.

Se define la cadena de fallo como el conjunto secuenciado de causas y efectos que se presentan en un proceso de fallo. El esquema viene reflejado en la figura 3.

En ella, puede observarse que los factores de contingencia o condicionantes explican la aparición de las causas últimas. Ambos (factores y causas) pueden confundirse, por lo que se señalarán los factores de contingencia (producto a procesar, alimentación de los equipos y máquinas, otros equipos o instalaciones conectadas, ambiente externo, etc.) como aquellos relativos al entorno del elemento, conjunto, máquina o equipo o sistema objeto, mientras que las causas se referirán a aspectos o elementos internos estructurales o funcionales.

Las causas primeras originan o promueven otras causas intermedias hasta llegar a las inmediatas al fallo, que normalmente son observables directamente. La relación entre las causas inmediatas y el fallo suele ser muy directa, con lo que a veces parece completar el 
análisis causa-efecto, sin proceder al descubrimiento de las causas últimas o factores condicionantes, objetivos finales del diagnóstico definitivo y concluyente del fallo.

Tampoco la cadena del fallo se detiene en la identificación del fallo, sino que es preciso considerar, aguas abajo, los efectos o consecuencias del fallo (económicas, de seguridad, laborales, medioambientales o de sostenibilidad, catastróficas, de imagen, sociales, etc.).

Cualquier elemento de la cadena de fallo es observable e identificable a través de unos síntomas o manifestaciones diversas (vibración, ruido o zumbido, olor, calor o frío, aumento o disminución de la visibilidad, humo, humedad, polvo, abrasión o corrosión, desgaste, rotura, desprendimiento, etc.). Estos síntomas son claves a la hora de identificar la cadena.

Se han considerado como fases del proceso de detección las siguientes: observación de síntomas y manifestaciones, identificación, detección, delimitación y descripción.

En la fase de observación de los síntomas y manifestaciones del fallo se trata de percibir información, a través de la observación sensorial directa, de la experiencia, de los conocimientos teóricos previos, de la información registrada, y de la medición o verificación a través de pruebas y ensayos. El análisis de esa información permite la identificación previa y con cierta inmediatez del fallo. Se perciben ya algunos accidentes del fallo; como, por ejemplo, lugar, posición o elemento que soporta el fallo.

En la fase de detección se obtienen comprobaciones pertinentes y contrastables sobre el fallo, que se completan en las dos fases siguientes: en la de delimitación se determinan básicamente los límites en el cumplimiento de la especificación y el proceso de fallo, en la de descripción se investigan las circunstancias del fallo (qué, dónde, cuándo, etc.). 


\section{CONCLUSIONES}

El estudio y conocimiento del proceso y la cadena de fallo son partes fundamentales en la mejora de los procesos que articulan la propia función de la empresa. La incertidumbre está unida intrínsicamente a los propios procesos (físicos y humanos), y su cuantificación e información permiten su acotación dentro de entornos controlables. Mediante la experimentalidad se puede mejorar el conocimiento de los propios procesos que limitan la incertidumbre y complementa a los estudios y modelos teóricos, que hacen mejorar la disponibilidad de la empresa o factoría. 


\section{REFERENCIAS BIBLIOGRÁFICAS}

Baeza, G., Rodríguez, P., Hernández, J. (2003). Evaluación de confiabilidad de sistemas de distribución eléctrica en desregulación. Revista Facultad de Ingeniería, Chile, 11 (1), pp. 33-39.

Bejar, J., (1974). Algunos modelos de mantenimiento. Novatécnia.

Beltrán, P., (1987). Mantenimiento predictivo de averías en máquinas rotativas. Objetivos, ventajas y justificación económica. Energía.

Beltrán, P., (1989). Aplicaciones de las técnicas de MPA a la recepción de maquinaria rotativa. Mantenimiento, Septiembre-Octubre.

British Standars, (1964). Glossary of General Terms used in maintenance Organization. (BS 3811:1964).

Cacique, J. (2007). Diseño de un programa para calcula la confiabilidad en un sistema de distribución de energía eléctrica. Trabajo Especial presentado como requisito parcial para optar al título de Ingeniero Electricista. UNEXPO. Venezuela, pp. 138.

Delgado, C. y García de la Fuente, M., (1994). Mantenimiento y sistemas expertos. Mantenimiento, Mayo-Junio.

Gómez de León, F. (1998). Tecnología del mantenimiento industrial. Servicio publicaciones de la Universidad de Murcia.

IEEE Std 493-2007 (2007). IEEE Recommended Practice for the Design of Reliable Industrial and Commercial Power Systems. Approved 7 February 2007. IEEE-SA Standards Board.

Koval, D., Zhang, X., Prost, J., Coyle, T., Arno, R., Hale, R. (2003). Reliability methodologies applied to the IEEE Gold Book standard network, IEEE Industry Applications Magazine, 9 (1), pp. 32-41.

Levitin G, Podofillini L, Zio E. (2003). Generalized importance measures for multi-state elements based on performance level restrictions. Reliability Engineering and System Safety 82, pp. 287-298.

López, M., Crespo, A., (2010). Modelling a Maintenance Management Framework Based on PAS 55 Standard. Qual. Reliab. Engng. Int., 27, pp. 805-820.

McGranaghan, M. (2007). Quantifying Reliability and Service Quality for Distribution Systems. IEEE Trans. Industry Applications, 43, pp. 188-195.

Ramos, G., Torres, A., (2007). Análisis de Confiabilidad de Sistemas Industriales Aplicando Redes Bayesianas Considerando Aspectos de PQ y Seguridad -Caso de Estudio Sistema IEEE 493. leee latin america transactions, 5 (8), pp. 605-610.

Sexto, L. (2005). Confiabilidad integral del activo. Seminario Internacional de Mantenimiento celebrado en Perú-Arequipa-Tecsup del 23-25 de febrero de 2005. 
Vásquez, C. Montesinos, M. Osal, W. y Blanco, C. (2007). Índices de Confiabilidad de Líneas Aéreas de Distribución. IV Simposio Internacional sobre la Calidad de la Energía Eléctrica SICEL2007. Ciudad de Medellín, Colombia.

Wang, W., Loman, J., Arno, R., Vassiliou, P., Furlong, E., Ogden, D., (2004). Reliability block diagram simulation techniques applied to the IEEE std. 493 Standard Network. IEEE Trans. Industry Applications, 40, pp. 887-955.

Weber, P., Jouffe, L. (2006). Complex system reliability modelling with dynamic object oriented Bayesian network, Reliability Engineering and System Safety, 91, pp. 149-162.

Yañez, M., Gómez de la Vega, H., Valbuena, G. (2003). Ingeniería de Confiabilidad y Análisis Probabilístico de Riesgo. ISBN 980-12-0116-9.

Yongli, Z., Limin, H., Jinling, L. (2006). Bayesian network-based approach for power systems fault diagnosis. IEEE Trans. on Power Delivery, 21 (2), pp. 634-639. 